\title{
ON OPERATORS ON SEPARABLE BANACH SPACES WITH ARBITRARY PRESCRIBED POINT SPECTRUM
}

\author{
GERHARD K. KALISCH ${ }^{1}$
}

\begin{abstract}
For every compact subset $C$ of $\boldsymbol{R}$ and every $p$ in $(1, \infty)$ there exists a bounded linear operator acting in a suitable closed subspace of $L_{p}(0,1)$ whose spectrum and point spectrum coincide with each other and with $C$.
\end{abstract}

The purpose of this note is to exhibit certain separable Banach spaces (including Hilbert space) that admit bounded linear operators with the property that their spectra $\sigma$ are pure point spectra with $\sigma$ any prescribed compact subset of the reals $\boldsymbol{R}$.

Consider $L_{p}(0,1)=L_{p}$ with $1<p<\infty$. Let $(M f)(x)=x f(x)$ and $(J f)(x)=\int_{0}^{x} f(y) d y$ for $f \in L_{p}$.

THEOREM. Let $C$ be a compact subset of $\boldsymbol{R}$. Then for every $p$ satisfying $1<p<\infty$ there exists a bounded linear operator $S$ acting in a closed subspace $\mathscr{K}$ of $L_{p}(0,1)$ whose spectrum coincides with its point spectrum and equals $C$. The operator $S$ may be chosen to be of the form $\alpha T_{1}+\beta$ with $\alpha>0$ and $\beta$ in $\boldsymbol{R}$ where $T_{1}$ is the restriction of $T=M-J$ on $L_{p}$ to a suitable closed invariant subspace $\mathscr{K}$ of $T$.

Proof. It suffices to show that if $C \subset[0,1)$, then there is an operator $T_{1}$ as described in the theorem with spectrum $T_{1}=$ point spectrum $T_{1}=C$. Let $\mathscr{K}$ be the closed subspace of $L_{p}$ spanned by $\Phi_{C}=\left\{\varphi_{\lambda} ; \lambda \in C\right\}$ where $T \varphi_{\lambda}=\lambda \varphi_{\lambda}$ and $\varphi_{\lambda}$ is the characteristic function of $[\lambda, 1]$. Clearly $\mathscr{K}$ is invariant under $T$; call $T_{1}$ its restriction to $\mathscr{K}$. The inclusion $C \subset$ point spectrum $T_{1}$ being immediate, we shall show that spectrum $T_{1} \subset C$ or rather complement $C \subset$ resolvent set of $T_{1}$, which proves the theorem.

Consider first $\zeta_{0} \notin[0,1]$. The formula $(M-J-\zeta)^{-1}=M_{t-\zeta}^{-1}+J M_{t-\zeta}^{-2}$ where $M_{g} f=g f$ shows that $\zeta_{0} \in$ resolvent set of $T$, so $\left(T-\zeta_{0}\right)^{-1}$ is a bounded operator on $L_{p}$. Let $\mathscr{K}_{0}$ be the set of all finite linear combinations of the $\varphi_{\lambda} \in \Phi_{C}$. We have $\left(T-\zeta_{0}\right)^{-1} \mathscr{K}_{0} \subset \mathscr{K}_{0}$ since

$$
\left(T-\zeta_{0}\right)^{-1} \sum \gamma_{j} \varphi_{j}=\sum\left(\lambda_{j}-\zeta_{0}\right)^{-1} \gamma_{j} \varphi_{j}
$$

Received by the editors December 4, 1970 and, in revised form, July 20, 1971. AMS 1970 subject classifications. Primary 47A10; Secondary 46C10, 46E30.

Key words and phrases. Bounded linear operator, $L_{p}$ spaces, point spectrum.

${ }^{1}$ I wish to acknowledge gratefully National Science Foundation Grant GP-21334.

(c) American Mathematical Society 1972 
where $\varphi_{j}=\varphi_{\lambda_{j}}$. Consider now $k=\lim k_{n} \in \mathscr{K}$ with $k_{n} \in \mathscr{K}_{0}$. We have $\left(T-\zeta_{0}\right)^{-1} k=\left(T-\zeta_{0}\right)^{-1} \lim k_{n}=\lim \left(T-\zeta_{0}\right)^{-1} k_{n} \in \mathscr{K}$ so that $\left(T-\zeta_{0}\right)^{-1} \mathscr{K} \subset$ $\mathscr{K}$. It is now an easy matter to check that the restriction of $\left(T-\zeta_{0}\right)^{-1}$ to $\mathscr{K}$ is an inverse of $T_{1}-\zeta_{0}$ so that $\zeta_{0} \in$ resolvent set of $T_{1}$.

If $\zeta_{0} \notin C$ but $\zeta_{0} \in[0,1]$ we can still show that $\left(T_{1}-\zeta_{0}\right)^{-1}$ exists as a bounded linear operator of $\mathscr{K}$ into itself so that in this case too we have $\zeta_{0} \in$ resolvent set of $T_{1}$. We first observe that if $I$ is an interval in the complement of $C$ in $[0,1]$, then the functions of $\mathscr{K}$, being limits of linear combinations of characteristic functions of $[\lambda, 1]$ with $\lambda \in C$, are constant on $I$. Consider then $\zeta_{0} \in I=\left(\lambda_{1}, \lambda_{2}\right) \subset[0,1] \backslash C$ and calculate $\left(T-\zeta_{0}\right)^{-1}$ on $\mathscr{K}$, as follows: every function $f_{0} \in \mathscr{K}$ can be written as $f_{0}=f_{1}+f_{2}$ with $f_{1}=f_{0}$ on the complement of $I, f_{1}=0$ on $I ; f_{2}=\gamma \chi_{I}$ where $\gamma$ is the constant value of $f_{0}$ on $I$. We have

with

$$
\left(T-\zeta_{0}\right)^{-1} f_{0}=\left(T-\zeta_{0}\right)^{-1} f_{1}+\left(T-\zeta_{0}\right)^{-1} f_{2}=g_{1}+g_{2}
$$

and

$$
g_{1}(t)=\left(t-\zeta_{0}\right)^{-1} f_{1}+\int_{0}^{t} \frac{f_{1}(s)}{\left(s-\zeta_{0}\right)^{2}} d s
$$

$$
\begin{gathered}
g_{2}(t)=0 \quad \text { on }\left[0, \lambda_{1}\right], \\
g_{2}(t)=\frac{-\gamma}{\zeta_{0}-\lambda_{1}} \quad \text { on } I=\left(\lambda_{1}, \lambda_{2}\right), \\
g_{2}(t)=\frac{-\gamma\left(\lambda_{2}-\lambda_{1}\right)}{\left(\lambda_{2}-\zeta_{0}\right)\left(\zeta_{0}-\lambda_{1}\right)} \text { on }\left[\lambda_{2}, 1\right] .
\end{gathered}
$$

This may be seen as follows:

(1) Observe that on $\mathscr{K}$, the operator $T-\zeta_{0}$ is $1-1$; otherwise there would exist a nonzero function $f \in \mathscr{K}$ such that $T f=\zeta_{0} f$ but the only functions with this property are multiples of $\varphi_{\zeta_{0}}$ which are not in $\mathscr{K}$ as the functions in $\mathscr{K}$ are constant on each interval in $[0,1] \backslash C$.

(2) We verify that $\left(T-\zeta_{0}\right)\left(g_{0}+g_{1}\right)=f_{1}+f_{2}=f_{0}$ by means of a simple calculation. We have $\left\|g_{j}\right\| \leqq M_{j}\|f\|$ where $M_{j}$ does not depend on $f_{0}$ but only on $\zeta_{0}$ and the $\lambda$ 's. Thus $\left(T-\zeta_{0}\right)^{-1}$ is bounded on $\mathscr{K}$. Since

$$
\left(T-\zeta_{0}\right)^{-1} \varphi_{\lambda}=\frac{1}{\lambda-\zeta_{0}} \varphi_{\lambda} \text { for } \lambda \neq \zeta_{0},
$$

we have $\left(T-\zeta_{0}\right)^{-1} \mathscr{K}_{0} \subset \mathscr{K}_{0}$ and we conclude as before that $\zeta_{0} \notin \operatorname{spectrum} T_{1}$. This concludes the proof of the theorem.

Department of Mathematics, University of California, Irvine, California 92664 